\title{
The pre-menstrual syndrome (PMS) treatment is unmasked, and it is simple and safe
}

\begin{abstract}
The Premenstrual Syndrome (PMS) is a very common problem for women who still menstruate but the prevention/treatment is not commonly understood. The surprise is that by treating the most common and vexing of the problems, facing these ladies, namely the excessive gas and bloating by an agent often prescribed for excessive gas leading to bloating, the troublesome features of PMS are also ameliorated. The agent recommended is the very safe and almost innocuous nystatin (Nilstat $\odot$, Mycostatin $\odot$ ), which acts by altering the permeability of the cell wall of intestinal yeast/fungi. There are hundreds of young ladies who can attest to the effectiveness of this method of helping them lead a PMS-free life.
\end{abstract}

Volume 2 Issue 3 - 2018

\author{
Charles H Pierce \\ Clinical Pharmacologist, USA
}

Correspondence: Charles H Pierce, Clinical Pharmacologist, Family Physician, Pharmacovigilance, and Regulatory Affairs, Cincinnati, Ohio, USA, Tel 5I3-508-6493,

Email charles.piercel@gmail.com

Received: February 07, 2018 | Published: June 15, 2018

Keywords: women, menstruate, innocuous nystatin, routine life, physicians, easy prevention, physical, emotional, syndrome, crying spells, panic attacks, insomnia, vaginitis

\section{Introduction}

By redressing a common problem in women that is usually not treated adequately, it is my hope that this will be viewed in a different light. I recommend that one think out of the box and with an open mind while reading this paper unmasking the quite safe and easy prevention / treatment of PMS. A good definition is: Premenstrual syndrome (PMS) refers to the cyclic occurrence of a set of disturbing physical, emotional or behavioral alterations that are of sufficient severity to interfere with interpersonal relations and routine life. ${ }^{1}$ Physicians need to know both the nature of this syndrome and how common it is in its many forms. Approximately 80 percent of ladies have pre-menstrual cramping due to the excess gas formation, which leads to the pain and bloating these girls and ladies experience. Also, the painful menstrual flow is noted for its different sized blood clots coupled with headaches, mood changes and occasionally nausea and vomiting. As if this isn't enough, some ladies have very life-disrupting symptoms including crying spells, panic attacks, and insomnia among other life interfering symptoms. Some label the most severe form of PMS the "Premenstrual Dysphoria Disorder (PMDD). No matter the severity, the elimination of the troublesome symptoms is the Goal. Any woman who has a period can get PMS, but some women are more likely to have symptoms than others:

\section{PMS is common in the late 20 s to mid-40s.}

II. Older teens tend to have more severe PMS.

III. PMS may be more severe in the 40 s.

IV. Women who've had at least one pregnancy are more prone

V. Women with a history of depression or other mood disorder may have more PMS symptoms.

\section{Diagnosis of this condition}

Over the last $40+$ of years of medical practice, I have found that those symptoms that show the highest frequency can be considered the main criteria and those that are less frequent can be considered the secondary criteria or hints that the patient to whom I am speaking may have PMS. The Main criteria that are common in patients with the "Pre-Menstrual Syndrome" are the following:

1. Gas and Bloating causing abdominal pain

2. Headaches and a general feeling of lousiness

3. Painful menstrual flow frequently with pieces of clotted blood The Secondary criteria, which are seen more variably and are usually refractory to standard medical therapies and which serve as clues or hints of the underlying problem, are the following:
a. Easy fatigability or loss of energy
b. Depression or lethargy
c. Feeling "strange", "unreal", or "spacey"
d. Irritability
e. Craving for sugar
f. Frequent fungal vaginitis, bouts of cystitis, or sinusitis type symptoms

g. A request for an anti-yeast pill after an anti/uncle biotic as they always get a "yeast infection" after these agents

Please note that the root cause or causes of this condition has not been clearly delineated. We do know that estrogen and progesterone drop the week before menstruation and some believe that this is a possible trigger for the symptoms. We also know that Birth Control medication does not eliminate the syndrome and is a generally ineffective treatment. ${ }^{1}$

\section{A real life story}

A typical story is that of A.S. who wrote "I have had a life-long struggle with heavy-flow, terrible cramps, migraines and nausea followed by vomiting. I spent some twenty-five years back and forth between doctors and specialist who put me on different medications including birth control, painkillers, and anti-depressants. Even after all of these drugs my symptoms persisted. I bloated and swelled so much 
that I purchased clothes and under-garments in bigger sizes, strictly for my cycle. My flow was so heavy that I needed to use a super plus absorbency tampon along with an overnight pad and still had issues with bleeding through onto my clothes. My headaches turned into migraines, my cramps and bloating led to constipation, which worsened uncomfortable pain. The older I became, the worse it was, and it began to occur more frequently. My cycle was unpredictable and at times would come twice a month." "I often joked that I only had one "good" week per month. Typically, would start PMS by getting very moody and emotional, which always accompanied a migraine. The next week was my actual period where I bled and bled some more. I passed numerous clots each day for up to seven days. The pieces of blood were at least quarter-sized or larger and always followed the type of cramps that often put me in the fetal position. After seeing many doctors who all tried to prescribe strong painkillers I finally gave in and began to take them, not knowing the after effect being on a narcotic for an extended period, could potentially lead. Years went past, and I continued on these treatments and still had zero results. I continued to bleed excessively and have intense migraines, vomiting, and bloating." "My life changed once I began taking nystatin. I have been able to track my periods accurately and plan accordingly. I have spent less money on pads and tampons and now have three "good" weeks in the month. I no longer have to wear larger clothes due to bloating and do not need to curl up in the fetal position and stay indoors. I feel incredible, and for the first time, I feel "normal." I only wish my doctors would have discovered this treatment 25 years ago, who knows what my life would have been. It is night and day from how I used to have to live. I am so happy with this treatment."

\section{Treatment of PMS}

The therapy of this problem called "PMS" is the antifungal/ antiyeast agent Nystatin (Nilstat $($, Mycostatin $($ ). The tablet form is readily available and most acceptable to patients. The tablets contain 500,000 units and are usually prescribed for two to three times a day starting 10 to 12 days before their expected menstruation and shortening the time pre-menses each successfully pain-free and symptom-free menstruation. I was aware of the use of the anti-yeast agent, Nystatin, for the management of painful gas and bloating from a professor of OBGYN while in Medical school. As he told us that he said that we should not be surprised that in addition to the expected reduction of intestinal gas that the painful periods that occurred in ladies with a condition known as "PMS" (Pre-Menstrual Syndrome) also disappeared. He told us this worked, but he did not have the answer as to how it worked. From Pharmacology, I knew that Nystatin was a very safe, almost innocuous agent, which was so poorly absorbed from the GI tract that it is considered non-absorbed and that it had a very enviably low side effect profile and is unquestionably safer than all other meds. Even young children get prescribed this agent for "Thrush", which is a condition of excess yeast in the child's mouth and throat. The only effect that I knew about was that it would suppress the growth of some fungi or yeast in the GI tract and decrease gas formation. ${ }^{2}$ I still do not know the mechanism by which nystatin ameliorates the broad spectrum of symptoms of PMS. These polyene antifungal agents have no other known effect other than altering the permeability of the fungal cell wall such that the organism cannot survive. This effect is not limited to the dimorphic Candida albicans but includes other hyphae forming organisms such as Penicillium and Aspergillus among others. ${ }^{3}$

\section{Summary}

It is indeed a pleasant experience to have patients return visibly gratified by the difference in how they feel when compared to how they felt before treatment. Few problems we treat in medicine are as rewarding or with medications that are as safe as Nystatin [2, 4]. This condition in women is far more frequent than you suspect. The goal is to use as little of any medication as possible, and every case must be individualized. ${ }^{5}$ The typical patient has been seen and unsuccessfully managed by Family Physicians, Internists, Allergists, and Gynecologists'. She has not been relieved of her painful menstruation coupled with gas and bloating (abdominal pain) and is desperate for help. Mainstream medicine seems to have invalidated a problem and its treatment just because they could not explain how or why it works. It would appear that thinking "out of the box" is not medicine's main suite. One of the main problems is that 'new uses' of older generic medications is hard to come by. The existing drug patent system is poorly designed to motivate discoveries of new uses for offpatent drugs. With the long histories, established safety records, and low prices of generic drugs, these products could be an effective way to improve health outcomes while lowering costs in new indications. ${ }^{6}$

\section{Acknowledgements}

None.

\section{Conflict of interest}

The author declares there is no conflict $\mathrm{f}$ interest.

\section{References}

1. Bakhshani NM, Hosseinbor M, Shahraki Z, et al. Premenstrual Syndrome Symptomatology among Married Women of Fertile Age based on Methods of Contraception (Hormonal versus Non-Hormonal Methods of Contraception. Glob J Health Sci. 2014;6(2):105-111.

2. Royal College of Obstetricians and Gynecologists. Management of premenstrual syndrome. 2007. p. 1-33.

3. O’Brien, PM, Backstrom, T, Brown C. Towards a consensus on diagnostic criteria, measurement and trial design of the premenstrual disorders: the ISPMD Montreal consensus. Arch Womens Ment Health. 2011;14(1):1321.

4. Yonkers KA, O’Brien PM, Eriksson E. Premenstrual syndrome. Lancet. 2008;371(9619):1200-1210.

5. Gillings MR. Were there evolutionary advantages to premenstrual syndrome? Evol Appl. 2014;7(8):897-904.

6. Sachs RE, Ginsberg PB, Goldman DP. Encouraging New Uses for old Drugs. JAMA. 2017318(24):2421-2422. 